\title{
Botulinumtoxin wirkt bei chronischer Migräne
}

Bisher wird Botulinumtoxin nicht zur Therapie der Migräne und des chronischen Kopfschmerzes empfohlen, denn in Studien war es Placebo nicht überlegen. Diese Empfehlung könnte sich durch die PREEMPT-Studie ändern.

S eit Anfang der 1990er-Jahre wird Botulinumtoxin als Therapie bei unterschiedlichen Kopfschmerzformen erprobt. In den Studien zur Prophylaxe der episodischen Migräne und des chronischen Kopfschmerzes vom Spannungstyp wurde schnell klar, dass Botulinumtoxin Placebo nicht überlegen war, sodass Botulinumtoxin für diese Indikationen nicht empfohlen werden kann. In einer 2007 veröffentlichten Studie zeigte sich jedoch in einer nachträglichen Analyse, dass die Untergruppe der Patienten mit chronischer Migräne von der Therapie mit Botulinumtoxin profitierte. Wissenschaftler stellen mit der hier vorliegenden Publikation die gepoolten Ergebnisse aus zwei großen, randomisierten, doppelblinden, placebokontrollierten Studien vor, in denen in Nordamerika und Europa 1.384 Patienten mit chronischer Migräne untersucht worden sind (PREEMPT-Studien).

Nach einer vierwöchigen ScreeningPhase wurde den Patienten entweder Placebo $(n=696)$ oder Onabotulinumtoxin A $(155-195 \mathrm{U})(\mathrm{n}=688)$ injiziert - dabei war Medikamentenübergebrauch (außer häufiger Opioidgebrauch) kein Ausschlusskriterium. Die Injektionen wurden alle 12 Wochen wiederholt. An eine 24 Wochen dauernde doppelblinde Phase schloss sich eine 32 Wochen dauernde Open-labelPhase an. Sowohl die Injektionsorte (Kopfund Nackenbereich) als auch die Dosis waren relativ streng festgelegt. In der vorliegenden Metaanalyse war der primäre Endpunkt die mittlere Frequenzänderung der Kopfschmerztage von Woche 20 bis 24 im Vergleich zum Ausgangspunkt, obwohl das nicht der primäre Endpunkt der PREEMPT-Studie 1 war.

In beiden Gruppen kam es zu einem deutlichen Rückgang der Kopfschmerztage: Unter Verum um 8,4 Tage und unter Placebo um 6,6 Tage. Der Unterschied zwischen beiden Gruppen war schon ab Woche vier nach Behandlung statistisch signifikant. Botulinumtoxin war zu allen Untersuchungszeitpunkten signifikant überlegen (ab Woche vier bis inklusive Woche 24) unter anderem auch in den sekundären Endpunkten „Frequenz der Migränetage“, „Tage mit mäßigen beziehungsweise starken Kopfschmerzen“, „Anzahl der kumulativen Stunden mit Kopfschmerzen an einem Kopfschmerztag“. In beiden Gruppen ging die Schmerzmitteleinnahme zur Akuttherapie zurück, jedoch ohne signifikanten Unterschied zwischen den Gruppen. Signifikant war aber die Reduktion der Kopfschmerztage: um mindestens $50 \%$ von $47,1 \%$ der Verumgruppe-Patienten und $35,1 \%$ in der Placebogruppe.

Dodick DW et al. Onabotulinumtoxin A for the treatment of chronic migraine: pooled results from the double-blind, randomized, placebocontrolled phases of the PREEMPT clinical program. Headache. 2010;50(6):921-36.

Kommentar von Prof. Stefan Evers: Gerade weil die chronische Migräne eine Migränekomplikation ist, die bisher nur unzureichend behandelt werden kann, ist das Ergebnis dieser Studien sowohl für den Patienten als auch den behandelnden Arzt erfreulich. Allerdings muss kritisch hinterfragt werden, ob der Unterschied zwischen Placebo und Verum klinisch relevant ist und diese Art von Therapie somit rechtfertigt. Inzwischen ist Botulinumtoxin in Deutschland zur Behandlung der chronischen Migräne zugelassen worden, aber unter Auflagen (andere Medikamente dürfen nicht wirksam gewesen beziehungsweise nicht vertragen worden sein, die Diagnose muss von einem Kopfschmerzexperten gestellt werden). Es bleibt abzuwarten, wie sich die Therapie mit Botulinumtoxin durchsetzt. Auf jeden Fall wäre ein gutes Register sinnvoll, in dem man die Daten der behandelten Patienten sammelt und analysiert, um so vielleicht doch noch eine Untergruppe zu identifizieren, die besonders gut von Botulinumtoxin profitiert.

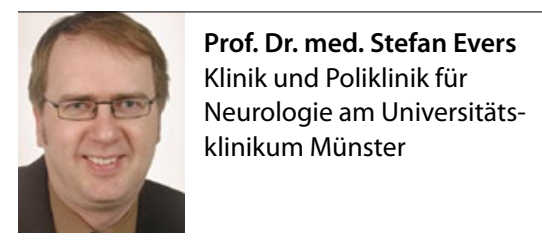

